\title{
Structural Characterization of Immune Responses to HPV Infection and HPV Vaccination
}

\author{
Suruchi Singh ${ }^{1}$, Jody Carter $^{1}$, Kirsten Cohen $^{1}$, Julie McElrath ${ }^{1}$, Denise Galloway ${ }^{1}$ and Marie Pancera ${ }^{1 *}$ \\ 1. Fred Hutchinson Cancer Research Center, Seattle, WA, USA. \\ * Corresponding author: mpancera@fredhutch.org
}

Human papillomavirus is the major cause of cervical cancer, which is the second most common cancer in women [1]. Three widely approved HPV vaccines that provide protection are a bivalent, a quadrivalent and a nonavalent vaccine [2]. All three vaccines elicit high titers of potent, type-specific antibodies (Abs) that prevent HPV infection. These Abs are directed against the major capsid protein, L1, from each HPV strains, which self-assembles to form virus-like particles (VLPs) that are the basis of the vaccine. The HPV vaccines induce a strong immune response against HPV16 and 18, as opposed to the weak immune response induced by HPV infections. Studies on the epitope specificity of anti-HPV human sera are complicated by the polyclonal nature of the response [3]. We currently have no understanding of the molecular features of human antibodies important for recognition of HPVs and of the differences between memory B cell responses. To understand about these differences and the type of immune response elicited at the molecular level, we aim to obtain atomic-level structural details of vaccine-elicited and natural infection-elicited antibodies from different B cell responses in complex with HPV16 L1 as pentamer or VLP.

Experimental Procedures: HPV-specific B cells were identified and sorted using fluorochrome-labeled HPV16 pseudoviruses by our collaborators following methods described previously [4,5]. The HPV16L1 protein was expressed in BL21(DE3) cells and the soluble protein was purified using GST agarose beads followed by size exclusion chromatography. Antibodies were expressed in mammalian HEK293EBNA cells and further purified using protein A agarose beads. For structural studies, the purified IgGs were digested to antigen binding fragment (Fab) using Lys $\mathrm{C}$ and further purified by SEC. To understand the epitope recognized by each antibody, we performed antigenicity characterization using ELISA. The sitting drop vapor diffusion method was used to crystallize L1-Fab complexes and unliganded Fabs. The nsEM grids of L1-Fab complexes were prepared to obtain 2D and 3D reconstruction of the complexes.

Results: Our collaborators studies on the Merck 9-valent HPV vaccine (GARDASILÒ9) induced B-cell response resulted in the molecular cloning of hmAbs (human monoclonal antibodies) from HPV16specific memory B-cells [4] and plasmablasts [5] and they determined that the majority of hmAbs require FG loop for neutralization. To further map the neutralizing epitopes and identify molecular features of hmAbs that corresponds to epitope specificity at the molecular level, we initially chose to study four antibodies for which the Vh genes were overrepresented (Table 1). We first successfully expressed monodisperse HPV16 L1 pentamers, with expected antigenic profile (shown by ELISA) (Fig. 1A). Second, we were able to form monodisperse complexes of HPV16 L1 with these antibodies by SEC (one is shown as example) (Fig. 1B). nsEM data also showed complexes formation with nice "star" shape (Fig. 1C). We have collected nsEM data for one of the protein complexes and trying to obtain 2D and $3 \mathrm{D}$ reconstruction. We are further planning to use these samples for single particle cryo-EM. We have also solved the crystal structures of unliganded Fabs (Fig. 1D) which will help in solving the structure of the complex using Cryo-EM. 
Conclusions: Since licensed vaccines for HPV can induce protective antibodies, we aim to understand the determinants of the antibody response, at a molecular level, and to compare these when induced by vaccination and natural infection. Moreover, the structural characterization of host immune response to an effective vaccine might shed light on specific mechanisms that could be applied to other pathogens for which vaccines do not exist or can be improved upon [6].

References:

[1] World Health Organization, Wkly Epidemiol Rec. 92.19 (2017), p. 241.

[2] DM Harper and LR DeMars, Gynecol Oncol. 146 (2017), p.196.

[3] JJ Orozco et al., J Virol. 79.15 (2005), p. 9503.

[4] EM Scherer et al., PLoS Pathog. 10.10 (2014), p. e1004461.

[5] EM Scherer et al., EBioMedicine 10 (2016), p. 55.

[6] The authors acknowledge VIDD internal funding and APS (Advanced Photon Source) beamline 19ID for X-ray diffraction data collection.

\begin{tabular}{|c|c|c|c|}
\hline & Heavy chain & Light chain & Epitope mapping \\
\hline 7nv08 & VH2-70 & VL1-40 & BC loop \\
\hline D24.1M01 & VH2-70 & VL1-40 & FGa loop \\
\hline E7nv03 & VH4-34, D3-16*02 & VL3-11 & FGb loop \\
\hline H16V5 & VH4-34, D3-16*02 & VL2-14 & FGb loop \\
\hline
\end{tabular}

Table 1. HPV16 L1-specific hmAbs characteristics
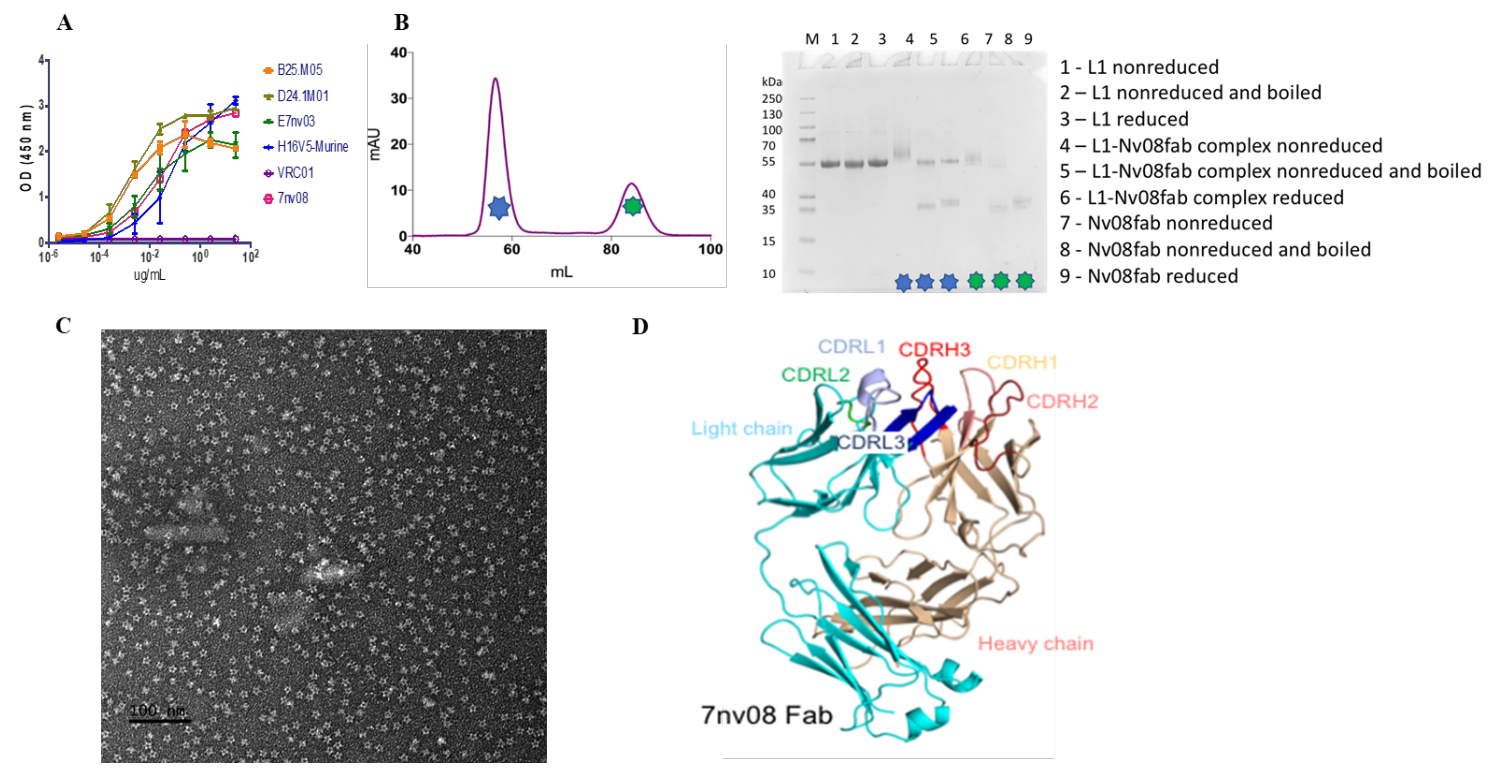

D

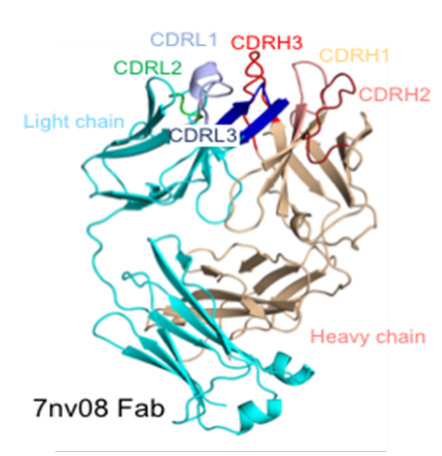

Figure 1. HPV16 L1 pentamer binding to vaccine-elicited hmAbs. A. ELISA showing binding of the hmAbs to the HPV16 L1 pentamer. B. SEC profile showing complex formation between HPV16 L1 pentamer and 7nv08 Fab and accompanying SDS-Page gel. C. nsEM of the complex (star shape) D. Cartoon representation of $7 \mathrm{nv} 08 \mathrm{Fab}$ at $2 \AA$ with combining determining regions (CDR) labeled and colored. 